\title{
Avaliação do aproveitamento do titânio contido nos minérios de nióbio da região de Catalão
}

Fernando Rodrigues da Cunha ${ }^{1}$ André Carlos Silva 2* (D)

\section{Resumo}

Os minérios de nióbio beneficiados na região de Catalão/GO contêm ilmenita e outros minerais de titânio que se concentram na etapa de flotação de pirocloro, dificultando o processo produtivo e contaminando o produto. A ilmenita, mais abundante e principal mineral-minério de titânio, é matéria-prima para a produção de rutilo sintético, escória de titânio e liga FeTi. Mais de $90 \%$ da produção mundial de titânio é direcionada para produção de dióxido de titânio, no entanto o processo aluminotérmico de produção de liga FeTi, possui as vantagens de ser adaptável à concentrados mais pobres, produzir metal livre de U e Th e ser uma tecnologia já bem estabelecida em Catalão para produção do $\mathrm{FeNb}$. $\mathrm{O}$ mercado de FeTi existe, inclusive com empresas comercializando no Brasil. A avaliação inicial do aproveitamento da ilmenita dos minérios de nióbio para produção da liga FeTi mostrou potencial para faturamento anual de até US\$ 96 milhões, mas são necessárias mais análises de grau de liberação e teor da ilmenita, além de ensaios de concentração em bancada e/ou piloto.

Palavras-chave: Ilmenita; FeTi; Nióbio; Titânio.

\section{Evaluation of the recovery of titanium contained in the niobium ores from Catalão region}

\begin{abstract}
The niobium ores processed in the region of Catalão/GO contain ilmenite and other titanium bearing minerals that concentrate on the pyrochlore flotation stage, making difficult the production process and contaminating the final product. Ilmenite, the most abundant and main titanium bearing mineral is the raw material for the synthetic rutile production, titanium slag, and FeTi alloy. More than $90 \%$ of the world's titanium production is used in the titanium dioxide production. However, the aluminothermy process used to produce $\mathrm{FeTi}$ alloy has the advantages of being adaptable to lower grade concentrates, producing a metallic alloy free of $\mathrm{U}$ and $\mathrm{Th}$, a procedure well established in Catalão for FeNb alloy production. The FeTi market exists with companies trading in Brazil. The initial evaluation of the ilmenite recovery from the niobium ores to produce FeTi alloy showed potential for annual revenues of up to US\$ 96 million. However, further mineral liberation analysis and ilmenite grade is required, as well as concentration tests in bench and/or pilot scale.
\end{abstract}

Keywords: Ilmenite; FeTi; Niobium; Titanium.

\section{Introdução}

A Niobras, empresa do grupo China Molybdenum (CMOC Brasil), é atualmente o segundo maior produtor mundial de liga ferronióbio (FeNb), com uma produção média de $9 \mathrm{kt} \mathrm{Nb} / a n o$. Situada entre os municípios de Catalão e Ouvidor, no estado de Goiás, teve a sua operação iniciada nos anos 70, com o início da produção do concentrado de pirocloro (mineral-minério de nióbio) em 1976 e da produção de liga $\mathrm{FeNb}$ em 1977. Na época o desenvolvimento do processo aluminotérmico se fez essencial, tendo em vista que o concentrado de nióbio $(\mathrm{Nb})$ produzido continha teores de urânio e tório que impediam sua exportação, o que não ocorre com a liga metálica produzida por este processo.

A lavra do minério de $\mathrm{Nb}$ foi iniciada pelas Minas I e II na região denominada Chapadão, fazendo divisa com

${ }^{1}$ Laboratório de Modelamento e Processamento Mineral, Universidade Federal de Goiás - UFG, Catalão, GO, Brasil.

${ }^{2}$ Universidade Federal de Catalão - UFCAT, Catalão, GO, Brasil.

*Autor correspondente: ancarsil@ufcat.edu.br

2176-1523 C 2021. Cunha et al. Publicado pela ABM. Este é um artigo publicado em acesso aberto (Open Access) sob a licença Creative Commons Attribution, que permite uso, distribuição e reprodução em qualquer meio, sem restrições desde que o trabalho original seja corretamente citado. 
as minas de fosfato. No final dos anos 90 e início de 2000, iniciou-se a operação da Mina Boa Vista, localizada no município de Catalão. Em 2008 teve início a operação da Planta Tailings (do inglês, rejeitos), que permitiu o aproveitamento do pirocloro contido nos rejeitos do processamento de rocha fosfática da Copebras (empresa pertencente ao grupo CMOC Brasil). Ao longo desses anos, as características do minério processado e, consequentemente, o processo produtivo, mudaram de acordo com o avanço da lavra e locais de jazimento. Contudo, os minerais portadores de titânio (Ti) sempre estiveram presentes no ROM (em inglês Run of Mine) como um contaminante do pirocloro.

Atualmente, a Niobras mantém três plantas de processamento mineral de $\mathrm{Nb}$ em operação, sendo elas a Tailings, a Boa Vista Fresh Rock (BVFR) e a Boa Vista (BV). As duas primeiras plantas processam minério oriundo da Mina Boa Vista. Contudo, a planta BV trabalha com minério das zonas oxidadas da mina e a planta BVFR com rocha fresca. O processo produtivo da liga FeNb na Niobras consiste basicamente nas seguintes etapas de lavra, processamento mineral e metalurgia. O processamento mineral é composto por britagem, separação magnética de baixa intensidade a seco (denominada localmente de escalpe), homogeneização, moagem primária e secundária (em circuito fechado com hidrociclones), separação magnética de baixa intensidade a úmido, deslamagem (hidrociclones) e flotação, que se divide em duas etapas de flotação reversa (flotação de carbonatos seguida pela flotação de silicatos) e uma direta de pirocloro. As etapas de metalurgia (hidro e piro) são compostas por lixiviação, calcinação e redução aluminotérmica. Ao final do processo a liga FeNb é submetida a uma etapa de britagem para adequação granulométrica do produto final, de onde o mesmo segue para embalagem e expedição. Os minerais portadores de Ti tendem a se concentrar na flotação de pirocloro, diminuindo o teor do concentrado final e dificultando o controle do processo aluminotérmico. Isto se deve ao fato de que as análises químicas de rotina (feitas por fluorescência de raios X) não permitem a identificação das fases minerais portadoras de Ti presente no concentrado final. Soma-se a isso o fato do modelo operacional (balanço de massas e energia) não estar preparado para essa entrada.

O Ti metálico e suas ligas possuem propriedades aplicáveis em tecnologias de ponta nas indústrias aeronáutica, espacial, naval, nuclear, química, automobilística, petróleo e gás, além de implantes, próteses e bens de consumo. No entanto, em 2004 95\% da produção mundial de matérias-primas de Ti foi destinada à produção de pigmento de dióxido de Ti, que é um material de altos índice de refração e alvura, alto poder de cobertura, opacidade, inércia química e toxicidade nula. Assim sendo, atualmente o principal uso do Ti é como um pigmento branco para tintas e revestimentos, borracha, papel e enchimento de reforço em plásticos. $\mathrm{TiO}_{2}$ também é usado em cerâmicas artificiais, tais como revestimentos para hastes de solda, como agregado pesado, fundentes de fornos de aço. Menos que $2 \%$ de todo Ti produzido é usado para a produção de Ti metálico e/ou suas ligas [1].
O Ti é utilizado na aciaria como redutor e elemento de liga, na forma de ferro-titânio (FeTi) ou sucata. Aços tratados com $\mathrm{Ti}$, ou contendo certas quantidades dele, normalmente possuem melhores propriedades mecânicas como resultado da efíciente desgaseificação e distribuição uniforme das inclusões de enxofre [2].

Segundo Bedinger [3], os principais minerais de Ti são a ilmenita $\left(\mathrm{FeO} . \mathrm{TiO}_{2}\right)$, o rutilo $\left(\mathrm{TiO}_{2}\right)$, o leucoxênio (que é um produto da alteração ou mistura de óxidos de $\mathrm{Fe}$ e Ti, incluindo titanita, ilmenita e perovskita, geralmente reconhecido como um rutilo ou anatásio em granulometria muito fina), o anatásio $\left(\mathrm{TiO}_{2}\right)$ e a perovskita $\left(\mathrm{CaTiO}_{3}\right)$, sendo que apenas os três primeiros minerais tem interesse econômico atualmente. Segundo Amorim e Almeida [4], a ilmenita é o mineral de Ti mais comum e abundante, sendo composto por até $53 \%$ de $\mathrm{TiO}_{2}$. Já o rutilo, de ocorrência menos comum que a ilmenita, é composto basicamente de $\mathrm{TiO}_{2}$, podendo conter até $10 \%$ de impurezas. No Brasil existem importantes ocorrências de anatásio, porém ainda sem nenhuma exploração industrial por falta de uma rota de processo economicamente viável para este mineral.

A Tabela 1 apresenta a estimativa de produções e reservas mundiais de ilmenita e rutilo por país (dados de 2018). Os recursos minerais mundiais de anatásio, ilmenita e rutilo quando combinados somam mais de 2 bilhões de toneladas [5]. É possível notar a predominância da disponibilidade da ilmenita como mineral minério de Ti. Os principais países produtores de ilmenita são Canadá, China, Austrália, Moçambique e África do Sul. Já os principais produtores de rutilo são Austrália, Serra Leoa, África do Sul, Ucrânia e Quênia. O Brasil detém aproximadamente $5 \%$ das reservas mundiais de ilmenita, porém apenas $1 \%$ da produção mundial.

A concentração de minerais portadores de Ti é geralmente realizada através de métodos gravíticos (ou densitários) e magnéticos (normalmente de alta intensidade). A ilmenita é frequentemente processada para a produção de escória sintética ou titanífera. Embora numerosas tecnologias sejam usadas para a produção de rutilo sintético, quase todas são baseadas em lixiviação seletiva ou redução térmica de Fe e outras impurezas presentes na ilmenita [3].

A flotação pode ser usada para a concentração de partículas finas, naturais ou não, atuando em faixas granulométricas inadequadas para os métodos anteriormente citados. A flotação da ilmenita e dos minerais oxidados de $\mathrm{Fe}$ pode ser realizada utilizando coletores carboxílicos (ácidos graxos e seus sabões), sulfonatos e aminas (tipicamente em $\mathrm{pH}$ acima do ponto isoelétrico do mineral a ser flotado) [6]. Contudo, ilmenita é caracterizada por apresentar baixa flotabilidade, resultando em baixas recuperação (mássica e/ou metalúrgica) $[7,8]$.

A liga FeTi pode ser produzida industrialmente por refusão de materiais reciclados, produzindo ligas de teores mais altos (por exemplo, 70Ti-30Fe), ou pela redução aluminotérmica da ilmenita, produzindo ligas de teores mais baixos (por exemplo, 25Ti-75Fe). Concentrados de ilmenita com $42 \%$ de $\mathrm{TiO}_{2}$ são suficientes para produção 
Tabela 1. Estimativa de produções e reservas mundiais de ilmenita e rutilo em 2018 [5]

\begin{tabular}{|c|c|c|c|c|c|c|c|c|}
\hline \multirow{3}{*}{ Países } & \multicolumn{4}{|c|}{ Produção } & \multicolumn{4}{|c|}{ Reservas } \\
\hline & \multicolumn{2}{|c|}{ Ilmenita } & \multicolumn{2}{|c|}{ Rutilo } & \multicolumn{2}{|c|}{ Ilmenita } & \multicolumn{2}{|c|}{ Rutilo } \\
\hline & $\left(10^{3} t\right)$ & $(\%)$ & $\left(10^{3} t\right)$ & $(\%)$ & $\left(10^{3} t\right)$ & $(\%)$ & $\left(10^{3} t\right)$ & $(\%)$ \\
\hline África do Sul & 500 & 9,3 & 100 & 13,3 & 63.000 & 7,2 & 8.300 & 13,4 \\
\hline Austrália & 700 & 13,0 & 250 & 33,3 & 250.000 & 28,4 & 29.000 & 46,8 \\
\hline Brasil & 50 & 0,9 & - & - & 43.000 & 4,9 & - & - \\
\hline Canadá & 850 & 15,7 & - & - & 31.000 & 3,5 & - & - \\
\hline China & 850 & 15,7 & - & - & 230.000 & 26,1 & - & - \\
\hline Estados Unidos $^{(1)}$ & $100^{(2)}$ & 1,9 & (2) & (2) & $2.000^{(2)}$ & 0,2 & (2) & (2) \\
\hline Índia & 300 & 5,6 & 10 & 1,3 & 85.000 & 9,7 & 7.400 & 11,9 \\
\hline Quênia & 280 & 5,2 & 90 & 12,0 & 54.000 & 6,1 & 13.000 & 21,0 \\
\hline Madagascar & 100 & 1,9 & - & - & 40.000 & 4,5 & - & - \\
\hline Moçambique & 600 & 11,1 & 8 & 1,1 & 14.000 & 1,6 & 880 & 1,4 \\
\hline Noruega & 200 & 3,7 & - & - & 37.000 & 4,2 & - & - \\
\hline Serra Leoa & - & - & 170 & 22,7 & - & - & 490 & 0,8 \\
\hline Senegal & 250 & 4,6 & 8 & 1,1 & ND & - & ND & - \\
\hline Ucrânia & 230 & 4,3 & 100 & 13,3 & 5.900 & 0,7 & 2.500 & 4,0 \\
\hline Vietnã & 200 & 3,7 & - & - & 1.600 & 0,2 & - & - \\
\hline Outros países & 150 & 2,8 & 10 & 1,3 & 26.000 & 3,0 & 400 & 0,6 \\
\hline Mundo (arredondamento) & 5.400 & 100,0 & 750 & 100,0 & 880.000 & 100,0 & 62.000 & 100,0 \\
\hline
\end{tabular}

Dados em $\mathrm{TiO}_{2}$ contido; (ND) Dado não disponível; (-) Dado não divulgado ou nulo; (1) Arredondado para as 100.000 toneladas mais próximas para evitar a divulgação de dados de propriedade da empresa; (2) Dados de produção e reservas de rutilo dos EUA estão incluídos com ilmenita.

de ligas com 29\% de Ti em sua composição [2]. Os óxidos de Fe e Ti são reduzidos em presença de alumínio conforme mostrado na reação (1), produzindo a liga metálica e uma escória à base de alumina.

$$
\mathrm{FeTiO}_{3}+2 \mathrm{Al} \rightarrow \mathrm{Ti}+\mathrm{Fe}+\mathrm{Al}_{2} \mathrm{O}_{3}
$$

A capacidade de produção anual mundial da liga FeTi é de aproximadamente 64.000 toneladas. Os principais produtores são a London \& Scandinavian Metallurgical em Rotterham, no Reino Unido ( $25 \mathrm{kt} / \mathrm{ano}$ ) e a VSMPO, na Rússia (16 kt/ano). Em 2006 os preços do médios da liga FeTi $(65,5 \mathrm{Fe}-30 \mathrm{Ti}-4,5 \mathrm{Al})$ comercializada na forma de lumps de $25 \mathrm{~kg}$ era de US\$5,7/kg (ou seja, US\$17/ $\mathrm{kg}$ de Ti contido na liga) [1].

$\mathrm{O}$ Ti forma ligas com o Fe em qualquer proporção, mas o diagrama de fases Fe-Ti apresenta 2 pontos eutéticos. O primeiro em $14 \%$ de Ti e o segundo em $68 \%$ de Ti [9]. Existem hoje quatro padrões comerciais da liga FeTi, baseados em seus teores de Ti, C, Si e Al, conforme apresentado na Tabela 2 [10]. No entanto, algumas empresas comercializam no Brasil ligas com especificações diferentes, tais como a Star Ligas (25-30\% Ti) e a Comercial Cometa (25-35\% Ti).

As últimas publicações da Agência Nacional de Mineração (ANM) mostram uma tendência à importação de produtos de Ti de maior valor agregado, enquanto pequenas quantidades de produtos de baixo valor agregado são exportados. Não há registros de produção interna da liga FeTi, sendo que o país importou cerca de 3.000 toneladas anuais a um preço de aproximadamente US\$ $4 / \mathrm{kg}$ (vide Tabela 3). Os principais municípios produtores de Ti são Mataraca/PB, Santa Bárbara de Goiás/GO e São Francisco de Itabapoana/RJ. Apenas três empresas processavam Ti
Tabela 2. Especificações químicas das ligas FeTi comerciais [10]

\begin{tabular}{lcccc}
\hline \multicolumn{1}{c}{ Elemento } & Grau A & Grau B & Grau C & Grau D \\
\hline Ti (\%) & $65-75$ & $65-75$ & $35-45$ & $15-25$ \\
C (\% máx.) & 0,15 & 0,20 & 0,15 & 5,00 \\
Si (\% máx.) & 0,25 & 0,25 & 5,00 & 5,00 \\
Al (\% máx.) & 0,50 & 5,00 & 8,00 & 8,00 \\
\hline
\end{tabular}

Tabela 3. Volumes de produtos de Ti comercializados pelo Brasil e seus respectivos preços [11]

\begin{tabular}{|c|c|c|c|c|c|}
\hline & Produto & Unid. & $2013^{(\mathrm{r})}$ & $2014^{(\mathrm{r})}$ & $2015^{(p)}$ \\
\hline \multirow[t]{2}{*}{ Produção } & $\begin{array}{l}\text { Concentrado de } \\
\text { Ilmenita }\end{array}$ & $(\mathrm{t})$ & 78.264 & 81.278 & 78.991 \\
\hline & $\begin{array}{l}\text { Concentrado de } \\
\text { Rutilo }\end{array}$ & $(\mathrm{t})$ & 2.021 & 1.834 & 1.984 \\
\hline \multirow[t]{4}{*}{ Importação } & $\begin{array}{l}\text { Minérios de Ti } \\
\text { e concentrados }\end{array}$ & $(\mathrm{t})$ & 23.929 & 19.867 & 33.488 \\
\hline & Liga FeTi & $(\mathrm{t})$ & 2.245 & 2.933 & 3.212 \\
\hline & Obras de $\mathrm{Ti}$ & $(\mathrm{t})$ & 334 & 339 & 412 \\
\hline & $\begin{array}{l}\text { Pigmentos } \\
\left(>80 \% \text { de } \mathrm{TiO}_{2}\right)\end{array}$ & $(\mathrm{t})$ & 139.102 & 135.633 & 114.723 \\
\hline \multirow[t]{4}{*}{ Exportação } & Ilmenita & $(\mathrm{t})$ & 49.691 & 66.028 & 16.619 \\
\hline & Liga FeTi & $(\mathrm{t})$ & 57 & 127 & 31 \\
\hline & Obras de Ti & $(\mathrm{t})$ & 5 & 4 & 13 \\
\hline & $\begin{array}{l}\text { Outros } \\
\text { Pigmentos - } \\
\text { Dióxido de } \\
\text { Titânio }\end{array}$ & $(\mathrm{t})$ & 6.073 & 6.024 & 5.539 \\
\hline \multirow[t]{4}{*}{$\operatorname{Preços}^{(1)}$} & $\begin{array}{l}\text { Minérios de Ti } \\
\text { e concentrados }\end{array}$ & $(\mathrm{US} \$ / \mathrm{t})$ & 1.177 & 621 & 530 \\
\hline & Liga FeTi & $(\mathrm{US} \$ / \mathrm{t})$ & 4.613 & 4.288 & 3.771 \\
\hline & Obras de $\mathrm{Ti}$ & (US\$/t) & 108.924 & 122.133 & 116.715 \\
\hline & $\begin{array}{l}\text { Pigmentos } \\
(>80 \% \text { de } \\
\left.\mathrm{TiO}_{2}\right)\end{array}$ & $(\mathrm{USS} / \mathrm{t})$ & 2.805 & 2.503 & 2.244 \\
\hline
\end{tabular}

(1) Preço médio: comércio exterior base importação; ${ }^{\left({ }^{(}\right)}$Revisado; ${ }^{\left({ }^{(}\right)}$ Preliminar. 
no Brasil em 2015, a Cristal Mineração do Brasil Ltda., a Indústrias Nucleares do Brasil S.A. e a Titânio Goiás Mineração Indústria e Comércio Ltda. A Cristal Mineração do Brasil, empresa pertencente ao grupo internacional Cristal Global, segundo maior produtor mundial de $\mathrm{TiO}_{2}$, foi responsável por mais de $80 \%$ da produção nacional de Ti beneficiado [11].

\section{Materiais e métodos}

Amostras da alimentação, rejeito da separação magnética, concentrado da flotação de carbonatos e silicatos, e concentrado e rejeito da flotação de $\mathrm{Nb}$ foram coletadas na planta BVFR da Niobras no intervalo de 3 dias de operação das plantas (entre 01 e 03/12/2017). Da planta Tailings foram coletadas amostras da alimentação e do concentrado e rejeito da flotação de $\mathrm{Nb}$. As amostras coletadas foram submetidas a etapas de secagem em estufa, homogeneização e quarteamento de modo a obter alíquotas representativas de $1 \mathrm{~kg}$ cada, massa essa adequada às análises subsequentes. Análises de grau de liberação, definido como a porcentagem de uma espécie mineral que ocorre como partícula livre em relação ao somatório de partículas mistas e livres desta fase [12], associação mineral e granuloquímicas foram realizadas no Laboratório de Caracterização Tecnológica (LCT) da Escola Politécnica da Universidade de São Paulo (USP).

A avaliação da forma de ocorrência das fases minerais presentes nas amostras foi efetuada de forma automatizada, em seções polidas, utilizando o software Mineral Liberation Analyser (MLA) da FEI, acoplado a um microscópio eletrônico de varredura Quanta 650 FEG da FEI e sistema de microanálise por EDS Esprit da Bruker. As análises mineralógicas e associações minerais realizadas consideraram tanto o contraste de número atômico como a composição química de cada fase mineral, utilizando imagens de elétrons retroespalhados (BSE) e espectros de raios X característicos por EDS. Todos os produtos gerados no decorrer deste trabalho foram submetidos à análises químicas quantitativas por espectrometria de fluorescência de raios X (FRX) usando um espectrômetro AXIOX MAX série DY 5001 da PANalytical, operando com pastilhas fundidas. A perda ao fogo (PF) foi efetuada por gravimetria a $1.020^{\circ} \mathrm{C}$ por $2 \mathrm{~h}$.

\section{Resultados e discussão}

As análises químicas por FRX indicaram resultados interessantes com relação aos teores de Ti, elementos de terras raras, Th e $\mathrm{Zr}$, conforme mostrado na Tabela 4. A amostra de concentrado de $\mathrm{Nb}$ da planta Tailings apresentou teor de $\mathrm{Nb}_{2} \mathrm{O}_{5}$ de aproximadamente $40 \%$, valor esse $76,9 \%$ menor que o concentrado produzido na planta BVFR (aproximadamente 52\%). O concentrado de $\mathrm{Nb}$ da planta Tailings apresentou teores próximos a $10 \%$ para $\mathrm{TiO}_{2} \mathrm{e}$ $\mathrm{ZrO}_{2}, 2,0 \%$ de $\mathrm{ThO}_{2}$ e 4,7\% de óxidos de terras raras, ou simplesmente OTR (aproximadamente 2, $0 \%$ de $\mathrm{CeO}_{2} 1,0 \%$

Tabela 4. Resultados das análises químicas das amostras coletadas nas plantas BVFR e Tailings

\begin{tabular}{|c|c|c|c|c|c|c|c|c|c|}
\hline \multirow{2}{*}{$\begin{array}{c}\text { Planta } \\
\text { Teor }\end{array}$} & \multicolumn{6}{|c|}{ BVFR } & \multicolumn{3}{|c|}{ Tailings } \\
\hline & Alim & Rej mag & $\begin{array}{c}\text { Conc flot } \\
\text { carb }\end{array}$ & $\begin{array}{l}\text { Conc flot } \\
\text { silic }\end{array}$ & $\begin{array}{c}\text { Rej flot } \\
\text { Nb }\end{array}$ & $\begin{array}{c}\text { Conc flot } \\
\mathrm{Nb}\end{array}$ & Alim & $\begin{array}{c}\text { Rej flot } \\
\text { Nb }\end{array}$ & $\begin{array}{c}\text { Conc flot } \\
\mathrm{Nb}\end{array}$ \\
\hline $\mathrm{Nb}_{2} \mathrm{O}_{5}$ & 1,06 & 0,22 & 0,75 & 0,41 & 0,43 & 51,90 & 0,50 & 0,41 & 39,90 \\
\hline $\mathrm{TiO}_{2}$ & 1,81 & 2,42 & 0,79 & 1,38 & 2,37 & 5,82 & 4,34 & 7,74 & 10,50 \\
\hline $\mathrm{SiO}_{2}$ & 21,60 & 1,05 & 11,40 & 42,30 & 18,00 & 5,28 & 42,40 & 32,20 & 1,87 \\
\hline $\mathrm{CaO}$ & 18,60 & 1,17 & 29,40 & 2,25 & 26,30 & 11,90 & 5,75 & 3,18 & 5,94 \\
\hline $\mathrm{Fe}_{2} \mathrm{O}_{3}$ & 15,30 & 91,50 & 7,33 & 14,80 & 7,59 & 3,96 & 34,70 & 43,30 & 5,65 \\
\hline $\mathrm{MgO}$ & 6,14 & 0,76 & 5,66 & 11,80 & 4,74 & 1,58 & 1,55 & 1,97 & 0,43 \\
\hline $\mathrm{K}_{2} \mathrm{O}$ & 4,35 & 0,13 & 1,94 & 9,56 & 3,61 & 1,31 & NA & $<0,10$ & 0,15 \\
\hline $\mathrm{Al}_{2} \mathrm{O}_{3}$ & 4,05 & 0,21 & 1,63 & 7,92 & 4,09 & 0,92 & 0,86 & 0,84 & 0,42 \\
\hline $\mathrm{BaO}$ & 2,15 & 0,11 & 3,22 & 0,44 & 1,87 & 0,81 & 0,66 & 0,74 & 5,92 \\
\hline $\mathrm{P}_{2} \mathrm{O}_{5}$ & 1,71 & 0,05 & 5,01 & 0,11 & 0,43 & 0,14 & 4,63 & 3,25 & 0,94 \\
\hline $\mathrm{SrO}$ & 1,33 & 0,07 & 2,09 & 0,12 & 1,24 & 1,35 & 0,24 & NA & 1,65 \\
\hline $\mathrm{SO}_{3}$ & 1,00 & 0,55 & 1,59 & 0,88 & 0,59 & 0,48 & NA & $<0,10$ & 0,08 \\
\hline $\mathrm{Na}_{2} \mathrm{O}$ & 0,44 & $<0,10$ & 0,66 & 0,35 & 0,33 & 5,47 & NA & NA & NA \\
\hline $\mathrm{MnO}$ & 0,22 & 0,17 & 0,26 & 0,15 & 0,30 & 0,06 & 0,61 & NA & NA \\
\hline $\mathrm{ZrO}_{2}$ & 0,08 & 0,03 & 0,05 & 0,05 & 0,05 & 0,57 & 0,33 & 0,62 & 9,70 \\
\hline $\mathrm{ThO}_{2}$ & 0,06 & 0,02 & 0,06 & 0,02 & 0,03 & 1,80 & NA & 0,07 & 2,05 \\
\hline $\mathrm{Ta}_{2} \mathrm{O}_{5}$ & NA & NA & NA & NA & NA & NA & NA & NA & 0,75 \\
\hline $\mathrm{UO}_{2}$ & NA & NA & NA & NA & NA & NA & NA & NA & 0,51 \\
\hline $\mathrm{PF}$ & 18,70 & GF & 24,80 & 6,44 & 27,40 & 4,28 & 3,25 & 4,20 & 5,16 \\
\hline OTR & 0,64 & 0,08 & 1,170 & 0,09 & 0,43 & 2,20 & 0,98 & 0,96 & 4,71 \\
\hline Fechamento & 99,24 & 98,54 & 97,81 & 99,07 & 99,80 & 99,83 & 100,80 & 99,48 & 96,33 \\
\hline
\end{tabular}

Dados de percentual em massa; OTR: Soma dos teores dos óxidos de terras raras; PF: Perda ao fogo; GF: Ganho ao fogo; NA: elemento não dosado na curva quantitativa. 
de $\mathrm{Nd}_{2} \mathrm{O}_{3}$ ). Já o rejeito dessa planta apresentou teor de $\mathrm{TiO}_{2}$ igual a $7,7 \%$. No tocante à alimentação das plantas, a planta Tailings apresentou teor de $\mathrm{TiO}_{2} 240 \%$ superior ao da planta BVFR $(4,3 \%$ e $1,8 \%$, respectivamente) o que explica os teores mais altos de Ti nos fluxos de saída desta planta. A planta BVFR apresentou a mesma tendência de enriquecimento de Ti, Th e OTR no concentrado de Nb, porém os teores foram menores que do os da planta Tailings. Isso deveu provavelmente aos menores na alimentação da planta BVFR. Nota-se que os teores de Zr na planta BVFR foram consideravelmente mais baixos que na planta Tailings, principalmente quando comparados os concentrados.

A Tabela 5 apresenta a análise mineralógica das amostras. É possível perceber que no concentrado de $\mathrm{Nb}$ da planta Tailings grande parte do $\mathrm{Ti}, \mathrm{Zr}$ e $\mathrm{Th}$, assim como uma fração do $\mathrm{Nb}$ e do Ce estavam na forma de zirkelita $\left((\mathrm{Ca}, \mathrm{Th}, \mathrm{Ce}) \mathrm{Zr}(\mathrm{Ti}, \mathrm{Nb})_{2} \mathrm{O}_{7}\right)$. Este mineral possui de composição química teórica de $32,19 \% \mathrm{ZrO}_{2}, 31,30 \% \mathrm{TiO}_{2}, 17,36 \% \mathrm{Nb}_{2} \mathrm{O}_{5}$, $8,79 \% \mathrm{CaO}, 6,90 \% \mathrm{ThO}_{2}$ e $4,29 \% \mathrm{Ce}_{2} \mathrm{O}_{3}$ [13]. Apenas $20 \%$ do $\mathrm{TiO}_{2}$ estava na forma de ilmenita, anatásio e perovskita. Este resultado sugere que uma redução significativa do teor de Ti no concentrado de $\mathrm{Nb}$ da planta Tailings por operações de processamento mineral é difícil, ou mesmo impossível, visto que a maior parte do Ti estava na fase zirkelita, que contém cerca de $10 \%$ do $\mathrm{Nb}$ do concentrado, sendo uma fonte considerável deste elemento. Segundo o diagrama de Ellingham, espera-se que a maior parte do $\mathrm{Nb}$ e do $\mathrm{Ti}$ contidos na zirkelita serão reduzidos e incorporados à liga $\mathrm{FeNb}$, enquanto que os demais elementos ( $\mathrm{Zr}, \mathrm{Ca}$, Th e $\mathrm{Ce}$ ) não serão reduzidos e farão parte da escória.

No caso do concentrado de $\mathrm{Nb}$ da planta BVFR, a remoção da ilmenita contida nele $(2,3 \%)$ provocaria a redução de mais de um $1 \%$ do teor de $\mathrm{TiO}_{2}$. Os fluxos potencialmente mais interessantes para a produção de Ti seriam o rejeito da flotação de $\mathrm{Nb}$ da planta Tailings (com $13 \%$ de ilmenita) e o rejeito da flotação de $\mathrm{Nb}$ da planta BVFR (com 3\% de ilmenita).

A concentração de minerais é baseada nas diferenças de propriedades físicas e/ou físico-químicas entre os minerais que se deseja separar, sendo a sua performance dependente de fatores tais como a intensidade das diferenças supracitadas, $\mathrm{o}$ grau de liberação das fases minerais e granulometria, dentre outros. Testes adicionais ainda se fazem necessários para a correta definição do grau de liberação da ilmenita.

Tabela 5. Composição mineralógica das amostras estudadas

\begin{tabular}{|c|c|c|c|c|c|c|c|c|c|}
\hline \multirow{2}{*}{$\begin{array}{c}\text { Planta } \\
\text { Mineral }\end{array}$} & \multicolumn{6}{|c|}{ BVFR } & \multicolumn{3}{|c|}{ Tailings } \\
\hline & Alim & Rej mag & $\begin{array}{c}\text { Conc flot } \\
\text { carb }\end{array}$ & $\begin{array}{c}\text { Conc flot } \\
\text { silic } \\
\end{array}$ & $\begin{array}{c}\text { Rej flot } \\
\text { Nb }\end{array}$ & $\begin{array}{c}\text { Conc flot } \\
\mathrm{Nb}\end{array}$ & Alim & $\begin{array}{c}\text { Rej flot } \\
\text { Nb }\end{array}$ & $\begin{array}{c}\text { Conc flot } \\
\mathrm{Nb}\end{array}$ \\
\hline Pirocloro & 2,1 & 0,4 & 0,9 & 0,8 & 0,5 & 87,0 & 0,7 & 0,6 & 65,0 \\
\hline Calcita & 23,0 & 1,0 & 1,8 & 33,0 & 36,0 & 0,1 & & - & - \\
\hline Anquerita & 8,1 & 0,7 & 1,9 & 10,0 & 12,0 & 0,5 & & - & - \\
\hline Dolomita & 4,8 & 0,4 & 1,1 & 6,3 & 7,6 & 0,2 & - & - & - \\
\hline Siderita & 1,3 & 2,5 & 0,4 & 1,0 & 1,4 & 0,4 & - & - & - \\
\hline Outros carbonatos (BaSr) & 2,9 & 0,1 & 0,3 & 6,6 & 2,3 & 0,1 & - & - & - \\
\hline Estroncianita & 1,2 & $<0,1$ & 0,1 & 2,9 & 0,9 & $<0,1$ & - & - & - \\
\hline Zirkelita & - & - & - & - & - & - & 0,5 & 1,2 & 27,0 \\
\hline Goethita & - & - & - & - & - & - & 14,0 & 25,0 & 1,1 \\
\hline K-feldspato & 15,0 & 0,3 & 24,0 & 3,2 & 19,0 & 2,4 & - & $<0,1$ & 0,1 \\
\hline Argilominerais & 22,0 & 0,9 & 61,0 & 17,0 & 9,1 & 3,9 & 2,4 & 1,0 & 0,1 \\
\hline Piroxênio/anfibólio & 1,5 & 0,2 & 1,5 & 1,1 & 1,8 & 0,2 & - & - & - \\
\hline Quartzo & 1,8 & 0,1 & 3,3 & 0,9 & 2,0 & 0,1 & 41,0 & 29,0 & 1,0 \\
\hline Ilmenita & 2,0 & 0,9 & 0,6 & 0,4 & 3,0 & 2,3 & 7,5 & 13,0 & 1,9 \\
\hline Rutilo & 0,4 & 0,4 & 0,3 & 0,3 & 0,7 & 1,1 & - & - & - \\
\hline Anatásio & - & - & - & - & - & - & - & 0,2 & 0,4 \\
\hline Perovskita & - & - & - & - & - & - & - & $\mathbf{0 , 1}$ & 0,7 \\
\hline Óxidos de ferro & 7,6 & 91,0 & 1,3 & 0,5 & 0,3 & 0,1 & 19,0 & 18,0 & 0,6 \\
\hline Óxido-FeMn & - & - & - & - & - & - & 0,9 & - & - \\
\hline Barita & 1,2 & $<0,1$ & 0,2 & 1,6 & 1,6 & $<0,1$ & - & - & - \\
\hline Monazita & - & - & - & - & - & - & 1,5 & 2,9 & 0,6 \\
\hline Apatita & 4,5 & 0,1 & 0,2 & 13,0 & 0,9 & 0,4 & 9,7 & 5,0 & 0,2 \\
\hline Al-fosfato & - & - & - & - & - & - & 1,9 & 2,8 & 0,8 \\
\hline Baddeleita & - & - & - & - & - & - & - & 0,6 & 1,1 \\
\hline Óxido-MnBa & - & - & - & - & - & - & 0,2 & 1,0 & 0,1 \\
\hline Pirita & 0,5 & 0,7 & 0,6 & 0,9 & 0,1 & 1,0 & - & - & - \\
\hline Outros & 0,6 & 0,2 & 0,3 & 0,7 & 0,5 & 1,1 & 1,0 & 0,3 & 0,1 \\
\hline Fechamento & 100,5 & 99,9 & 99,8 & 100,2 & 99,7 & 100,9 & 100,3 & 100,7 & 100,8 \\
\hline
\end{tabular}

Dados de percentual em massa. (-) Não detectado ou detectado em quantidade muito pequena e somado a "outros"; argilominerais = micas e clorita; óxidos de ferro $=$ magnetita e hematita. 
Contudo, as análises de grau de liberação realizadas para o pirocloro indicaram liberações maiores que $90 \%$ nos concentrados de ambas plantas e cerca de $7 \%$ em associações binárias. Este fato deve-se em grande parte à distribuição do tamanho de partículas $\left(d_{10}=24,72 \mu \mathrm{m}, d_{50}=64,00 \mu \mathrm{m}\right.$ e $d_{90}=220,43 \mu \mathrm{m}$ ). Já para os rejeitos da flotação de $\mathrm{Nb}$ foram encontradas cerca de $66 \%$ das partículas de pirocloro liberadas, aproximadamente $24 \%$ em associações binárias e $10 \%$ em associações de três ou mais minerais, com distribuiç̧ão de tamanho de partículas mais grosseiro $\left(d_{10}=41,04 \mu \mathrm{m}\right.$, $d_{50}=111,94 \mu \mathrm{m}$ e $\left.d_{90}=253,76 \mu \mathrm{m}\right)$. Analisando a composição mineralógica dos rejeitos das flotações de $\mathrm{Nb}$ das duas plantas nota-se que pelo menos três propriedades diferenciadoras podem ser utilizadas na produção de concentrado de Ti, sendo elas a massa específica, susceptibilidade magnética e condutividade elétrica.

Segundo Premaratne e Rowson [14], a ilmenita comumente ocorre alterada em misturas de $\mathrm{TiO}_{2}, \mathrm{FeO}$ e $\mathrm{Fe}_{2} \mathrm{O}_{3}$, com o teor de $\mathrm{TiO}_{2}$ aumentando de 49 para $75 \%$ à medida que o mineral oxida e o Fe é lixiviado pela água subterrânea. Testes em escala de bancada e/ou piloto simulando operações industriais de separação magnética, concentração gravítica, separação eletrostática e flotação dos minerais portadores de Ti ainda precisam ser realizados. É impossível prever a performance de um processo de concentração sem a realização dessas etapas, porém, para efeito de avaliação inicial do potencial do negócio. Contudo, uma ferramenta útil para a análise de risco é simular cenários, desde os mais conservadores aos mais otimistas. Com esse objetivo foi construída a Tabela $6 \mathrm{com}$ dados da operação atual fornecidos pela Niobras, resultados das análises mineralógicas e químicas. Três cenários foram simulados, variando-se a recuperação mássica de ilmenita e disponibilidade física da planta de produção de ilmenita. Devido à falta de informações sobre o grau de liberação e teor da ilmenita, o cenário pessimista foi traçado assumindo uma recuperação mássica de ilmenita de $35 \%$. Para o cenário otimista foi assumida uma recuperação mássica de 55\%.
Para a disponibilidade física da planta (DF), aqui entendida como o total de horas operadas pela mesma, foi assumida uma variação de 80 a $90 \%$, valores razoáveis para a indústria mineral. Foi considerado o somatório das plantas BVFR e BV para o processamento do minério oriundo da Mina Boa Vista, visto que ambas plantas têm processos similares.

Uma possibilidade é a venda de concentrado de ilmenita, como é feito por algumas minas brasileiras, a preços médios de US\$100,00 a US\$ 150,00 por tonelada, com teor de mínimo de $\mathrm{TiO}_{2}$ de $50 \%$. Porém, no caso do minério de Catalão e região, existe uma possibilidade real de que o concentrado de ilmenita contenha teores de $\mathrm{U}$ e Th que possa impedir a sua comercialização. Além disso, o concentrado de ilmenita trata-se de um produto com baixo valor comercial quando comparado à liga FeTi. Desta forma, uma opção viável seria fechar a cadeia produtiva, produzindo a liga FeTi via processo aluminotérmico. Processo este já adotado industrialmente pela Niobras para a produção da liga $\mathrm{FeNb}$ e que este processo produz liga metálica livre dos isótopos supracitados. No processo aluminotérmico, entre 70 a $75 \%$ do titânio contido na ilmenita é reduzido à sua forma metálica e incorporado à liga FeTi. O maior custo desse processo é com insumos, particularmente o Al metálico em pó. Estequiometricamente são necessárias 2 partes de Al para cada parte de Ti produzida, como pode ser visto na reação (1), ou seja, são necessários $2 \mathrm{~kg}$ de alumínio para produzir $1 \mathrm{~kg}$ de Ti contido na liga FeTi [2]. A nível de comparação, na produção industrial da liga $\mathrm{FeNb}$, relação $\mathrm{Al}: \mathrm{Nb}$ é aproximadamente 1:1. Este fato, aliado ao preço de venda da liga FeTi substancialmente menor que a liga $\mathrm{FeNb}$, pode reduzir a margem de lucro do negócio. Contudo, a produção da liga FeTi em paralelo à produção da liga $\mathrm{FeNb}$ permitirá a produção da nova liga sem a geração de custos adicionais de lavra e cominuição, reduzindo consideravelmente o custo de produção da mesma.

Tabela 6. Simulação da produção de ilmenita, FeTi e faturamento bruto anual

\begin{tabular}{|c|c|c|c|c|c|c|}
\hline \multirow{2}{*}{$\begin{array}{c}\text { Cenário } \\
\text { Planta }\end{array}$} & \multicolumn{2}{|c|}{ Pessimista } & \multicolumn{2}{|c|}{ Realista } & \multicolumn{2}{|c|}{ Otimista } \\
\hline & BVFR+BV & Tailings & BVFR+BV & Tailings & BVFR+BV & Tailings \\
\hline Taxa de alimentação (t/h) & 275 & 140 & 275 & 140 & 275 & 140 \\
\hline Dist. Mass. Rej. flot. Nb (\%) & 37 & 40 & 37 & 40 & 37 & 40 \\
\hline $\mathrm{TiO}_{2}$ rej. flot. $\mathrm{Nb}(\%)$ & 2,37 & 7,74 & 2,37 & 7,74 & 2,37 & 7,74 \\
\hline Ilmenita no rej. flot. $\mathrm{Nb}(\%)$ & 3,0 & 13,0 & 3,0 & 13,0 & 3,0 & 13,0 \\
\hline Ilmenita disponível (t/h) & \multicolumn{2}{|c|}{10} & \multicolumn{2}{|c|}{10} & \multicolumn{2}{|c|}{10} \\
\hline Recuperação de ilmenita (\%) & \multicolumn{2}{|c|}{35} & \multicolumn{2}{|c|}{45} & \multicolumn{2}{|c|}{55} \\
\hline $\mathrm{DF}(\%)$ & \multicolumn{2}{|c|}{80} & \multicolumn{2}{|c|}{85} & \multicolumn{2}{|c|}{90} \\
\hline Ilmenita (t/ano) & \multicolumn{2}{|c|}{25.344} & \multicolumn{2}{|c|}{34.621} & \multicolumn{2}{|c|}{44.804} \\
\hline Preço ilmenita (US\$/t) & \multicolumn{2}{|c|}{100} & \multicolumn{2}{|c|}{125} & \multicolumn{2}{|c|}{150} \\
\hline Venda ilmenita (US\$/ano) & \multicolumn{2}{|c|}{ 2.534.356 } & \multicolumn{2}{|c|}{ 4.327.638 } & \multicolumn{2}{|c|}{6.720 .568} \\
\hline Rec. met. aluminot. (\%) & \multicolumn{2}{|c|}{65} & \multicolumn{2}{|c|}{70} & \multicolumn{2}{|c|}{75} \\
\hline Ti na liga FeTi (t/ano) & \multicolumn{2}{|c|}{5.230} & \multicolumn{2}{|c|}{7.694} & \multicolumn{2}{|c|}{10.668} \\
\hline Preço de venda (US\$/t Ti) & \multicolumn{2}{|c|}{7.000} & \multicolumn{2}{|c|}{8.000} & \multicolumn{2}{|c|}{9.000} \\
\hline Vend. FeTi (US\$/ano) & \multicolumn{2}{|c|}{36.608 .475} & \multicolumn{2}{|c|}{61.550 .513} & \multicolumn{2}{|c|}{96.011 .042} \\
\hline
\end{tabular}




\section{Conclusões}

O principal mineral portador de Ti presente no minério de $\mathrm{Nb}$ da região de Catalão é a ilmenita, apesar de existir ocorrência de Ti disseminado em outras fases minerais. Os rejeitos das flotações de $\mathrm{Nb}$ foram os fluxos analisados que contém maiores teores de ilmenita ( $3,0 \%$ da planta BVFR e 13,0\% da planta Tailings). Em termos de aproveitamento do Ti, estes fluxos são de grande interesse por tratar-se de um material sem nenhuma utilização atual, que é enviado diretamente para a barragem de rejeitos. $\mathrm{O}$ aproveitamento industrial da ilmenita presente nos rejeitos das flotações de $\mathrm{Nb}$ tem potencial de reduzir em mais de $30.000 \mathrm{t} / \mathrm{ano}$ de material destinado às barragens da Niobras.

Contudo, ensaios de concentração da ilmenita contida nos rejeitos em escala de bancada e/ou piloto se mostram necessários de modo a validar os cenários propostos. Teoricamente, as principais propriedades diferenciadoras entre a ilmenita e as demais fases minerais de ganga são a susceptibilidade magnética, massa específica e condutividade elétrica. Ainda além, não pode-se descartar a possibilidade do emprego de etapas de flotação.

No tocante à produção da liga $\mathrm{FeTi}$, este produto tem potencial para se tornar um novo negócio, com faturamento anual entre US\$ 37 milhões a US\$ 96 milhões. A produção de FeTi, através do processo aluminotérmico, se mostra uma alternativa interessante e viável para o aproveitamento da ilmenita que atualmente é enviada para a barragem de rejeitos. Tal processo, capaz de produzir uma liga FeTi isenta de $\mathrm{U}$ e $\mathrm{Th}$, já é realizado industrialmente pela Niobras. Desta forma não existiria a necessidade de aquisição de equipamentos ou insumos diferentes dos que atualmente são empregados na produção da liga $\mathrm{FeNb}$, o que tem potencial para reduzir o investimento através do compartilhamento das instalações industriais.

\section{Agradecimentos}

Os autores gostariam de agradecer à empresa Niobras/ CMOC por permitir a realização desta pesquisa, CNPq pelo financiamento do projeto, ao LaMPPMin e à UFCat.

\section{Referências}

1 Cardarelli F. Materials handbook: a concise desktop reference. 2nd ed. London: Springer-Verlag; 2008.

2 Riss M, Khodorovsky Y. Production of ferroalloys. Moscow: Foreign Languages Publishing House; 1963.

3 Bedinger GM. Titanium: minerals yearbook. Reston: USGS; 2018.

4 Amorim AA No, Almeida ABL. Titânio. In: Brasil. Economia mineral do Brasil. Brasília: Departamento Nacional de Produção Mineral; 2009.

5 Bedinger GM. Titanium: mineral commodity summaries 2019. Reston: USGS; 2019.

6 Baltar CAM, Cunha ASF. Aproveitamento de finos de ilmenita por flotação com succinamato. In: Anais do XX Encontro Nacional de Tratamento de Minérios e Metalurgia Extrativa; 2004; Florianópolis. Vol. 2. Belo Horizonte: UEMG; 2004. p. 51-59.

7 Zhong K, Cui L. Influence of $\mathrm{Fe}^{2+}$ ions of ilmenite on its flotability. International Journal of Mineral Processing. 1987;20(3-4):253-265.

8 Fan X, Rowson NA. The effect of $\mathrm{Pb}\left(\mathrm{NO}_{3}\right)_{2}$ on ilmenite flotation. Minerals Engineering. 2000;13(2):205-221.

9 Infomet. Diagrama de fases Fe-Ti. 2019 [acesso em 12 jul. 2019]. Disponível em: https://www.infomet.com.br/site/ diagrama-de-fases-ver.php?cod $=246$

10 COMETAL. Ferro titânio: generalidades. 2018 [acesso em 12 jul. 2018]. Disponível em: http://www.cometalsa.com/ productos-y-servicios/ferroaleaciones-en-piedra/2\#ancla14

11 Queiroz AA Fo, Amorim AA No. Titânio: sumário mineral. Brasília: Departamento Nacional de Produção Mineral; 2016.

12 Gaudin AM. Principles of mineral dressing. London: McGraw Hill; 1939.

13 Webmineral. Zirkelite Mineral Data. 2019 [acesso em 12 jul. 2019]. Disponível em: http://webmineral.com/data/ Zirkelite.shtml\#.XSjVvuhKjIU

14 Premaratne WAPJR, Rowson NA. Recovery of Titanium from beach sand by physical separation. The European Journal of Mineral Processing and Environmental Protection. 2004;4(3):183-193.

Recebido em: 2 Ago. 2019

Aceito em: 30 Set. 2020 